\title{
Optimal design of particle dampers for structures with low first eigenfrequency under forced vibration
}

\author{
N. Meyer ${ }^{\dagger *}$ and R. Seifried ${ }^{\dagger}$ \\ $\dagger$ Institute of Mechanics and Ocean Engineering \\ Hamburg University of Technology \\ Eißendorfer Strasse 42, 21073 Hamburg, Germany \\ e-mail: n.meyer@tuhh.de, web page: www.tuhh.de/mum
}

\begin{abstract}
Lightweight designs are becoming increasingly important these days to reduce energy consumption and natural resources. However, a smaller weight typically causes a decrease in stiffness and non-negligible vibration amplitudes over a wide frequency range. One passive damping technique to reduce such vibrations is the use of particle dampers. Thereby containers attached to a vibrating structure or holes embedded in the vibrating structure are filled with granular material. Due to the structural vibrations, momentum is transferred to the granular material which interacts with each other. As a result, energy is dissipated by impacts and frictional phenomena between the particles. Particle dampers show several advantages when compared to other existing passive damping techniques. They may be insensitive to temperature and environmental conditions, do not necessarily add significant mass, and do not degrade in time. In literature it has been shown that they are at least as effective as other damping techniques, and the effectiveness is not restricted to a single frequency but exists over a broader frequency range [1].

Recently, the rolling attribute of spheres has been used to design efficient particle dampers for low amplitude vibrations. As long as the container's acceleration stays below the gravitational acceleration, this rolling effect can be used to damp horizontal vibrations efficiently. The description of the damper's energy dissipation is accurately possible using analytical formulas which are presented here. These analytical formulas could enable a systematic design and quick calculation of the frequency response function for various technical applications, like high-rise buildings or wind power plants.

In this presentation, the workflow for such a systematic damper design is presented using the analytical formulas and validated experimentally. The analytical formulas are coupled to a modal reduced model of the utilized structure. An analytical expression for an optimal damper design is derived. Also, the calculation scheme to obtain the frequency response function of the system is presented. As an application example a simple beam-like structure is used, see figure 1. Its base point is subjected to a harmonic motion of variable frequency using a linear drive. The particle damper is mounted at the tip of the beam and its velocity is measured using a laser scanning vibrometer. Thus, the frequency response function is obtained. A good agreement between analytical and experimental obtained frequency response function is achieved for the optimized damper, validating the presented approach.
\end{abstract}
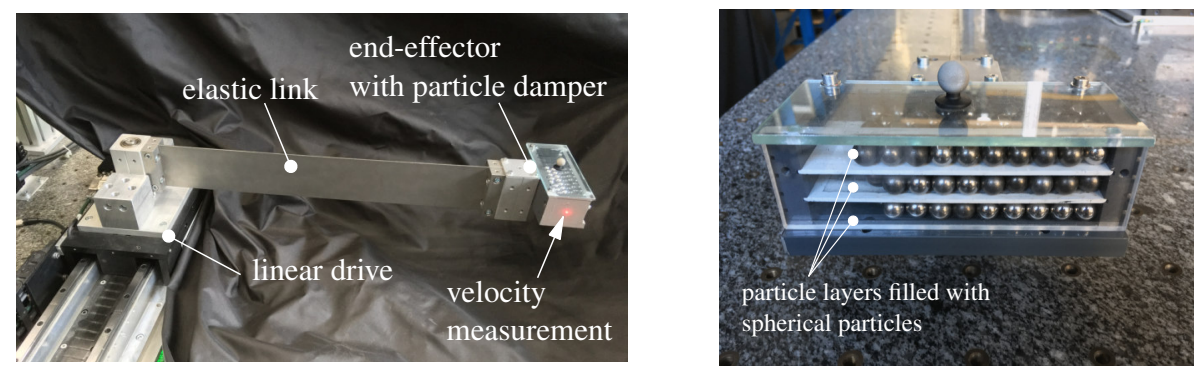

Figure 1: Simple beam-like structure. Left: Overview. Right: Augmentation of end-effector.

\section{REFERENCES}

[1] Panossian, H. Structural Damping Enhancement Via Non-Obstructive Particle Damping Technique, Journal of Vibration and Acoustics, vol. 105, no. 114, 1992. 